\title{
A RANK THEOREM FOR COHERENT ANALYTIC SHEAVES
}

\author{
BY \\ GÜNTHER TRAUTMANN( $\left.{ }^{1}\right)$
}

\begin{abstract}
Let $S$ be an analytic subvariety in $C^{n}$ and $\mathscr{F}$ a coherent analytic sheaf on $C^{n}$, such that $\mathscr{F}$ is locally free on $C^{n}-S$ and $\Gamma(U, \mathscr{F})=\Gamma(U-S, \mathscr{F})$ for every open set $U \subset C^{n}$. It is shown that $\mathscr{F}$ is locally free everywhere, if codh $\mathscr{F} \geqq n-1$ and $\operatorname{dim} S+\operatorname{rank} \mathscr{F} \leqq n-2$.
\end{abstract}

In this note the following question is examined. Let $S$ be an analytic subvariety in $C^{n}$ and $V$ be a vector bundle on $C^{n}-S$. Is it true that $V$ is globally trivial if $\operatorname{dim} S+\operatorname{rank} V \leqq n-2$ ? If we use results on the extension of coherent analytic sheaves, we may reformulate the question as follows. Let $\mathscr{F}$ be a coherent analytic sheaf on $\boldsymbol{C}^{n}$ and assume that

(a) $\mathscr{F} \mid C^{n}-S$ is locally free,

(b) for any open subset $U \subset C^{n}, \Gamma(U, \mathscr{F}) \cong \Gamma(U-S, \mathscr{F})$,

(c) $\operatorname{dim} S+$ rank $\mathscr{F} \leqq n-2$.

Is then $\mathscr{F}$ locally free everywhere? In the case $\operatorname{dim} S=0$ an affirmative answer of this question was conjectured by Wolfgang Barth. We prove here that this conjecture is right for general $S$ if we make the additional assumption $\operatorname{codh} \mathscr{F}_{x} \geqq n-1$ for every $x \in S$.

It turned out that the above question is only local in nature and in addition purely algebriac, see $\S 1$. It is related to a conjecture on the number of generators of analytic modules. In $\$ 2$ we use a result of [3] and [4] to establish a canonical resolution of a certain ideal sheaf which describes the singularities of the sheaf in question, and derive from this result the theorem.

1. Let $X$ be a domain in $C^{n}, \mathscr{F}$ a coherent analytic sheaf on $X$ and $S \subset X$ an analytic subvariety. We denote by $\mathcal{O}$ the sheaf of holomorphic functions on $X$. By $\mathscr{R}_{s}^{i} \mathscr{F}$ we denote the analytic sheaf on $X$ defined by the presheaf $U \leadsto H^{i}(U-S, \mathscr{F})$. In general the $\mathscr{R}_{S}^{i} \mathscr{F}$ are no longer coherent, see [7]. The following result however was proved in [5] and [6].

1.1. $\mathscr{F}=\mathscr{R}_{S}^{0} \mathscr{F}$ and $\mathscr{R}_{S}^{i} \mathscr{F}=0$, for $1 \leqq i \leqq q$, if and only if $\operatorname{dim} S \cap S_{k+q+2}(\mathscr{F}) \leqq k$ for all $k$, where $S_{m}(\mathscr{F})$ denotes the analytic subvariety of all $x \in X$ for which

Received by the editors June 5, 1970.

AMS 1970 subject classifications. Primary 32L10; Secondary 13D05.

Key words and phrases. Coherent analytic sheaves, homological codimension, rank, resolutions of ideals.

( $\left.{ }^{1}\right)$ During research for this paper the author was a Fulbright Scholar at the University of Cincinnati. 
$\operatorname{codh} \mathscr{F}_{x} \leqq m$. For every $x \in X$ we define rank $\mathscr{F}_{x}=\operatorname{dim}_{\mathscr{M}_{x}} \mathscr{M}_{x} \otimes_{\mathcal{O}_{x}} \mathscr{F}_{x}$ where $\mathscr{M}_{x}$ is the field of meromorphic function germs at $x$. Since $\mathscr{F}$ is coherent and $X$ connected rank $\mathscr{F}_{x}$ does not depend on $x$ and is denoted simply by rank $\mathscr{F}$.

1.2. The following three statements for a subvariety germ $S_{0}$ at $0 \in C^{n}$ are equivalent:

(1) Let $\mathscr{F}$ be a coherent analytic sheaf in a neighborhood $X$ of 0 in $C^{n}$, where $S_{0}$ has a representative $S$. If $\mathscr{F} \mid X-S$ is locally free, $\mathscr{F}=\mathscr{R}_{S}^{0} \mathscr{F}$, and $\operatorname{dim} S+\operatorname{rank} \mathscr{F}$ $\leqq n-2$, then $\mathscr{F}_{0}$ is free.

(2) Let $\mathscr{F}$ be a torsion-free coherent analytic sheaf in a neighborhood $X$ of 0 in $C^{n}$ where $S_{0}$ has a representative $S$. Let $\operatorname{codh} \mathscr{F}_{x} \geqq n-1$ for any $x \in X-S$. If then codh $\mathscr{F}_{0}<n-1$, we obtain for the minimal number $\mu=\mu\left(\mathscr{F}_{0}\right)$ of generators of $\mathscr{F}_{0}$ the estimate $\mu>(n-2)+\operatorname{rank} \mathscr{F}-\operatorname{dim} S$.

(3) Let $M$ be a noetherian module over $\mathcal{O}_{0}$ without torsion and let $\mathfrak{A} \subset \mathcal{O}_{0}$ be the ideal of $S_{0}$. If, for every prime ideal $\mathfrak{P} \ngtr \mathfrak{A}, \operatorname{codh} M_{\mathfrak{P}} \geqq n-1$ and codh $M<n-1$, then $\mu(M)>(n-2)+\operatorname{rank} M-\operatorname{dim} \mathfrak{A}$.

Proof. It follows by standard arguments that (2) and (3) are equivalent.

(1) $\Rightarrow(2)$. By shrinking $X$ we may assume that $\mathscr{F}$ has a representation $0 \rightarrow \mathscr{K} \rightarrow \mathscr{O}^{\mu} \rightarrow \mathscr{F} \rightarrow 0$ and rank $\mathscr{K}=\mu-$ rank $\mathscr{F}$. Since $\mathscr{F}$ is torsion-free, we have $\mathscr{K}=\mathscr{R}_{S}^{0} \mathscr{K}$. Also $\mathscr{K} \mid X-S$ is locally free. Since, however, codh $\mathscr{K}_{0}$ $=\operatorname{codh} \mathscr{F}_{0}+1<n, \mathscr{K}_{0}$ is not free. Hence by (1) rank $\mathscr{K}+\operatorname{dim} S>n-2$ which gives the estimate.

$(2) \Rightarrow(1)$. Let $\mathscr{F} *$ denote the sheaf $\operatorname{Hom}_{\mathcal{O}}(\mathscr{F}, \mathcal{O})$. By a representation $\mathscr{O}^{g} \rightarrow \mathscr{F}^{*} \rightarrow 0$ we get an exact sequence $0 \rightarrow \mathscr{F}^{* *} \rightarrow \mathcal{O}^{g} \rightarrow \mathscr{G} \rightarrow 0$ where $\mathscr{G}$ is without torsion. Now since $\mathscr{F} \mid X-S$ is locally free, we obtain $\mathscr{F}=\mathscr{R}_{S}^{0} \mathscr{F}=\mathscr{R}_{S}^{0} \mathscr{F} * *$. Since however $\mathscr{F}^{* *}$ is reflexive, also $\mathscr{R}_{S}^{0} \mathscr{F} * *=\mathscr{F} * *$. Hence we have the exact sequence $0 \rightarrow \mathscr{F} \rightarrow \mathcal{O}^{g} \rightarrow \mathscr{G} \rightarrow 0$. Now $\mu\left(\mathscr{G}_{0}\right) \leqq g$, and since rank $\mathscr{G}=g-$ rank $\mathscr{F}$ we get $\mu\left(\mathscr{G}_{0}\right) \leqq(n-2)+\operatorname{rank} \mathscr{G}-\operatorname{dim} S$ by the assumptions in (1). By (2) $\operatorname{codh} \mathscr{G}_{0} \geqq n-1$, and hence $\mathscr{F}_{0}$ is free.

2. Let $z_{11}, \ldots, z_{1 n} ; z_{21}, \ldots, z_{2 n} ; \ldots ; z_{p 1}, \ldots, z_{p n}$ be the $p n$ coordinates in $C^{N}$, $N=p n$ and let $p \leqq n$. Denote by $D_{v_{1} \ldots v_{p}}$ the determinants of the matrix $\left(z_{i v_{j}}\right)_{1 \leqq i, j \leqq p}$ where $1 \leqq \nu_{1}<\cdots<\nu_{p} \leqq n$. Let further $\mathscr{D}_{p}(n)$ be the ideal sheaf on $C^{N}$ generated by the holomorphic functions $D_{v_{1} \ldots v_{p}}$. It is proved in [3] and [4] that codh $\mathscr{D}_{p}(n)$ $\geqq N-(n-p)$ and that $S_{p}(n)=\operatorname{Supp}\left(\mathcal{O} / \mathscr{D}_{p}(n)\right)$ is a perfect analytic subvariety of pure dimension $N-(n-p)-1$.

2.1. It can be shown moreover that $\mathscr{D}_{p}(n)$ has a canonical resolution

$$
0 \rightarrow \mathcal{O}^{g_{n-p}} \rightarrow \cdots \rightarrow \mathcal{O}^{g_{k}} \rightarrow \cdots \rightarrow \mathcal{O}^{g_{1}} \rightarrow \mathcal{O}^{g_{0}} \rightarrow \mathscr{D}_{p}(n) \rightarrow 0
$$

with $g_{k}=\left({ }^{p+k-1}\right)\left(\begin{array}{c}n \\ p+k\end{array}\right)$, which is some kind of a generalized Koszul-complex, see [2]. One can construct this resolution inductively and thereby obtain the result of Northcott. By this, one can also show that all the matrices representing the homomorphisms in this sequence are in terms of the coordinates $z_{i j}$. 
2.2. Let now $\left(a_{i j}\right)_{1 \leqq i \leqq p ; 1 \leqq j \leqq n}$ be a matrix of holomorphic functions in a domain $X$ in $C^{M}$. Let $\rho: X \rightarrow C^{N}$ be the holomorphic mapping defined by $z_{i j}=a_{i j}$. If $A_{v_{1} \ldots v_{p}}$ denote the subdeterminants of order $p$ of $\left(a_{i j}\right)$ we have $A_{v_{1} \ldots v_{p}}=D_{v_{1} \ldots v_{p}} \circ \rho$. Then $\mathscr{A}=\rho^{*} \mathscr{D}_{p}(n)$ is the ideal sheaf generated by the functions $A_{v_{1} \ldots v_{p}}$. We prove now

2.3. Let $S$ be the subvariety of $\mathscr{A}$ in $X$. If $\operatorname{dim} S \leqq M-(n-p)-1$ (this implies that $\left(a_{i j}\right)$ has maximal rank $\left.p\right)$ then $\mathscr{A}$ has a canonical resolution

$$
0 \rightarrow \mathcal{O}_{M}^{g_{n}-p} \rightarrow \cdots \rightarrow \mathcal{O}_{M}^{g_{k}} \rightarrow \cdots \rightarrow \mathcal{O}_{M}^{g_{0}} \rightarrow \mathscr{A} \rightarrow 0
$$

with $g_{k}=\left(\begin{array}{c}p+k-1 \\ k\end{array}\right)\left(\begin{array}{c}n \\ p+k\end{array}\right)$. Especially $S$ has pure dimension $M-(n-p)-1$ and is perfect.

Proof. Let $\mathcal{O}_{M}$ (resp. $\left.\mathcal{O}_{N}\right)$ denote the structure sheaves on $X$ (resp. $\left.C^{N}\right)$. Let $\mathscr{Z}^{i}=\operatorname{Im}\left(\mathcal{O}_{N}^{g_{i}} \rightarrow \mathcal{O}_{N}^{g_{i-1}}\right)$ and $\mathscr{Z}^{n-p}=\mathcal{O}_{N}^{g_{n-p}}, \mathscr{Z}^{0}=\mathscr{D}_{p}(n)$. By 2.1 we have the exact sequences

$$
0 \rightarrow \mathscr{Z}^{i+1} \rightarrow \mathcal{O}_{N}^{g_{i}} \rightarrow \mathscr{Z}^{i} \rightarrow 0, \quad 0 \leqq i \leqq n-p-1 .
$$

We define $\mathscr{A}^{i}=\rho^{* \mathscr{Z}^{i}} \otimes_{\rho^{*} \mathcal{O}_{N}} \mathcal{O}_{M}$ where $\mathcal{O}_{M}$ is considered a $\rho^{*} \mathcal{O}_{N}$-module by the sheaf morphism $\rho * \mathcal{O}_{N} \rightarrow \mathcal{O}_{M}$ induced by $\rho$. We obtain the exact sequences

$$
\mathscr{A}^{i+1} \rightarrow \mathcal{O}_{M}^{g_{i}} \rightarrow \mathscr{A}^{i} \rightarrow 0
$$

and

$$
0 \rightarrow \mathscr{A}^{i+1}\left|X-S \rightarrow \mathscr{O}_{M}^{g}\right| X-S \rightarrow \mathscr{A}^{i} \mid X-S \rightarrow 0
$$

since $\rho(S)=S_{p}(n)$ and $S=\rho^{-1}\left(S_{p}(n)\right)$ and since $\rho^{*} \mathscr{D}_{p}(n)_{x}=\mathscr{D}_{\rho}(n)_{\rho(x)}$ is a free $\rho^{*}\left(\mathcal{O}_{N}\right)_{x}=\left(\mathcal{O}_{N}\right)_{\rho(x)}$-module for $x \notin S$. From these we obtain the following diagrams:

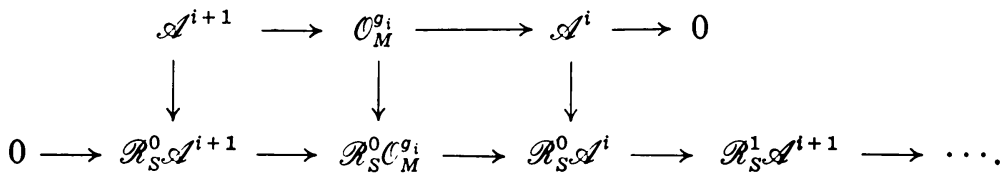

Using the result 1.1 we can prove by induction that $\mathscr{R}_{S}^{0} \mathscr{A}^{i}=\mathscr{A}^{i}$ for $1 \leqq i \leqq p-n$ and $\mathscr{R}_{S}^{j} \mathscr{A}^{i}=0$ for $1 \leqq j<i$. Hence all the sequences $0 \rightarrow \mathscr{A}^{i+1} \rightarrow \mathscr{O}_{M}^{g_{i}} \rightarrow \mathscr{A}^{i} \rightarrow 0$ are exact and 2.3 has been proved.

3. From 2.3 the main result follows now very easily.

3.1. TheOREM. Let $X$ be a neighborhood of 0 in $C^{m}$ and $S$ a subvariety in $X$. Assume $\mathscr{F}$ is a coherent analytic sheaf on $X$ having a representation $0 \rightarrow \mathcal{O}^{p} \rightarrow \mathcal{O}^{n}$ $\rightarrow \mathscr{F} \rightarrow 0$. If $\mathscr{F} \mid X-S$ is locally free, $\mathscr{F}=\mathscr{R}_{S}^{0} \mathscr{F}$ and $\operatorname{dim}_{0} S+$ rank $\mathscr{F} \leqq m-2$, then $\mathscr{F}_{0}$ is free.

Proof. Let $\left(a_{i j}\right)$ be the matrix by which $\alpha$ is determined. $\left(a_{i j}\right)$ has maximal rank. Let $\mathscr{A}$ be as in $\S 2$. Then Supp $(\mathscr{O} / \mathscr{A}) \subset S$ since $\mathscr{F} \mid X-S$ is locally free, by a wellknown fact. Hence $\operatorname{dim} \operatorname{Supp}(\mathcal{O} / \mathscr{A}) \leqq m-2-(n-p)$ because $n-p=\operatorname{rank} \mathscr{F}$. By 
2.3 , codh $\mathscr{A} \geqq m-(n-p)$ and, by $1.1, \mathscr{A}=\mathscr{R}_{S}^{0} \mathscr{A}=\mathcal{O}$ and hence $\operatorname{Supp}(\mathscr{O} \mid \mathscr{A})=\varnothing$. Hence $\mathscr{F}_{0}$ is free.

3.2. EXAMPLE. Let $\mathscr{T}$ be the ideal sheaf of the subvariety

$$
A=\left\{z_{1}=z_{2}=0\right\} \cup\left\{z_{3}=z_{4}=0\right\}
$$

in $C^{4}\left[5\right.$, p. 86]. For $x \in A-\{0\}$ we have codh $\mathscr{T}_{x}=3$ and, since $\mathscr{R}_{00\}}^{0} \mathscr{T}=\mathscr{T}$, $\operatorname{codh} \mathscr{T}_{0} \geqq 2$ by 1.1. Since however $\mathscr{R}_{\{0\}}^{0}(\mathcal{O} \mid \mathscr{T}) \neq \mathcal{O} \mid \mathscr{T}$, we have codh $\mathscr{T}_{0}=2$ also by 1.1. Hence

$$
\begin{aligned}
\operatorname{codh} \mathscr{T}_{x} & =4, & & x \notin A, \\
& =3, & & x \in A-\{0\}, \\
& =2, & & x=0 .
\end{aligned}
$$

Let $\mu\left(\mathscr{T}_{0}\right)$ be the minimal number of generators of $\mathscr{T}_{0}$. By (1) $\Rightarrow(2)$ in 1.2 , we obtain $\mu>(4-2)+1+0=3$ for $S=\{0\}$; on the other hand, $z_{1} z_{3}, z_{1} z_{4}, z_{2} z_{3}, z_{2} z_{4}$ are four generators of $\mathscr{T}_{0}$. Hence $\mu\left(\mathscr{T}_{0}\right)=4$.

\section{REFERENCES}

1. M. Auslander and D. A. Buchsbaum, Codimension and multiplicity, Ann. of Math. (2) 68 (1958), 625-657. MR 20 \#6414.

2. D. A. Buchsbaum, A generalized Koszul complex. I, Trans. Amer. Math. Soc. 111 (1964), 183-196. MR 28 \#3075.

3. J. A. Eagon and D. G. Northcott, Ideals defined by matrices and a certain complex associated with them, Proc. Roy. Soc. Ser. A 269 (1962), 188-204. MR 26 \#161.

4. D. G. Northcott, Some remarks on the theory of ideals defined by matrices, Quart. J. Math. Oxford Ser. (2) 14 (1963), 193-204. MR 27 \#1467.

5. G. Scheja, Fortsetzungssätze der komplex-analytischen Cohomologie und ihre algebraische Charakterisierung, Math. Ann. 157 (1964), 75-94. MR 31 \#738.

6. G. Trautmann, Eine Bemerkung zur Struktur der kohärenten analytischen Garben, Arch. Math. (Basel) 19 (1968), 300-304. MR 37 \#4288.

7. — Ein Endlichkeitssatz in der analytischen Geometrie, Invent. Math. 8 (1969), 143-174. MR 40 \#4482.

UNIVERSITY OF CINCINNATI, Cincinnati, OHIO 45221 